\title{
Plastic Deformation Behavior of Rail Steel under Cyclic Impact Blows
}

\author{
Makoto AKAMA \\ Senior Engineer, \\ Metallic Materials G., \\ Materials Technology Development Div., \\ Technological Development Dept.
}

\author{
Shinsaku MATSUYAMA \\ Chief Engineer, \\ Material Lab., \\ Technical Research laboratory, \\ Toyo Electric Manufacturing Co. Ltd.
}

\begin{abstract}
In order to demonstrate clearly the damaging mechanism of rail surfaces at the joint subjected to impact blows by train wheels, cyclic impact blow tests and low cycle fatigue tests have been performed using $0.75 \% \mathrm{C}$ rail steel with different hardness values and microstructures. The results are summarized as follows. (1) Under cyclic impact blows, cyclic softening occurs at an early deformation stage in tempered martensite, but only hardening occurs following surface cracking afterward in pearlite. (2) Under low cycle fatigue conditions, cyclic softening at a small strain amplitude turns to hardening as the strain amplitude increases in pearlite, although only cyclic softening occurs more extensively in tempered martensite. (3) The fact that dislocation structures formed under cyclic impact blows correspond to the structural changes observed under cyclic plastic straining suggests that the fatigue behavior under impact blows can be roughly estimated through low cycle fatigue tests.
\end{abstract}

Keywords : Rail steel, Cyclic impact blow, Low cycle fatigue, Cyclic hardening, Cyclic softening.

\section{Introduction}

The repeated impact blows given by a passing train cause plastic flow and sometimes horizontal cracking in the heads of rail ends at the joints, as shown in Fig. 1. In order to prevent this type of damage, End-Hardened rails (EH rails), which have a fine pearlite structure formed by slack quenching with hardness values ranging from HV320 to HV372 have come to be widely used.

Although the effectiveness of the $\mathrm{EH}$ rail has been confirmed by field tests, greater durability has come to

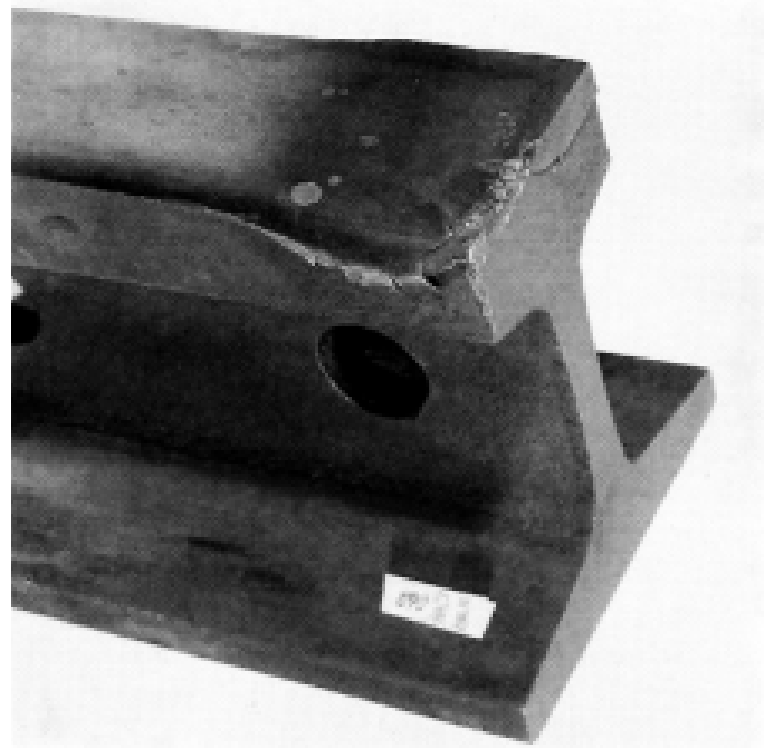

Fig. 1 A crushed head at rail end be required of rails with the increase in train speed and frequency. Furthermore, Head-Hardened rails (HH rails) and New-type Head Hardened rails (NHH rails) also have come to be used for the purpose of decreasing the gauge corner wear of outside rails.

On the other hand, the characteristics of plastic flow due to cyclic impact blows have not been clarified from a metallurgical viewpoint despite such rails being used for long years.

In this study, cyclic impact blow tests and low cycle fatigue tests have been performed using $0.75 \% \mathrm{C}$ rail steel with different hardness values and structures in order to throw light on the rail head crushing mechanism.

\section{Experimental procedures}

\section{1 Specimen and heat treatment}

Specimens were taken from the head of an as-rolled $\mathrm{NHH}$ rail. The chemical composition and specimen sizes are shown in Table 1 and Fig. 2, respectively. Specimens were heat-treated after machining (Table 2 ). In the case of pearlite structure, the hardness depends on inter-lamellar spacing, which becomes smaller with decreases in the pearlite-forming temperature. Therefore, the hardness level was controlled by isothermal transformation using a salt bath.

In the case of tempered martensite structure, on the other hand, the hardness level was controlled by the tem-

Table 1 Chemical composition of specimen (wt \%)

\begin{tabular}{ccccc}
\hline $\mathrm{C}$ & $\mathrm{Si}$ & $\mathrm{Mn}$ & $\mathrm{P}$ & $\mathrm{S}$ \\
\hline 0.75 & 0.25 & 0.93 & 0.022 & 0.006 \\
\hline
\end{tabular}


Table 2 Heat treatment of specimen

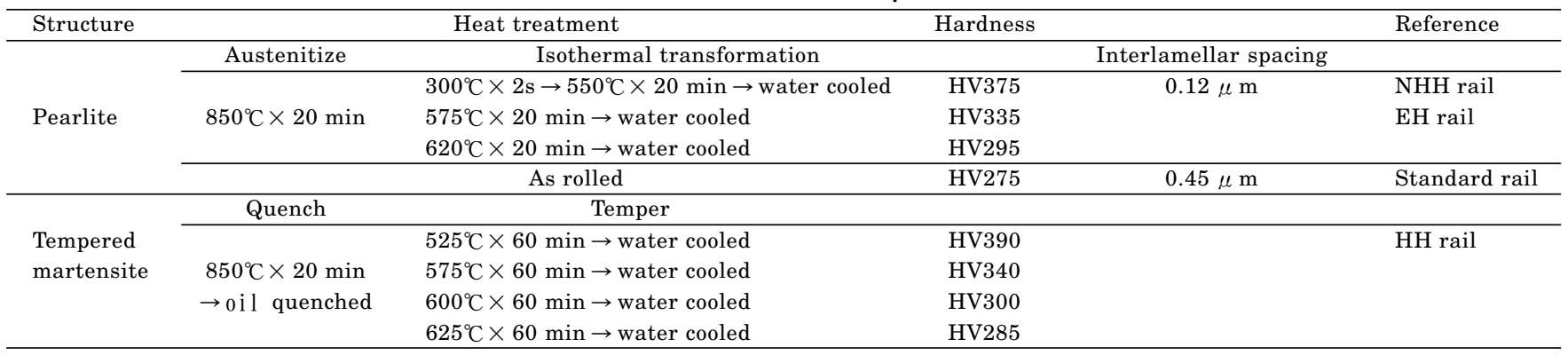

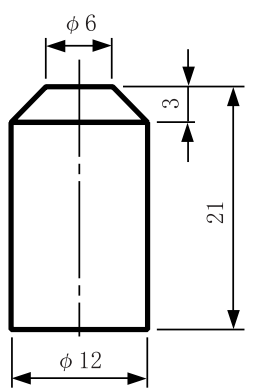

(a) Impact blow test

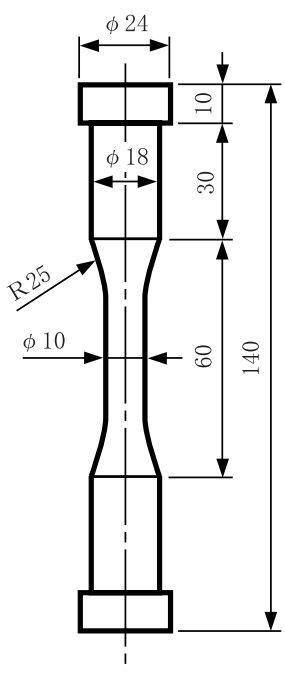

(b) Low cycle fatigue test

pering temperature.

\section{2 Cyclic impact blow test}

The cyclic impact blow test was performed using a Matsumura-type Impact Fatigue test machine (Fig. 3) with a modified impact hammer (HV500). Impact loads were measured by four strain gauges attached symmetrically with respect to the axis of the hammer at a distance of $10 \mathrm{~mm}$ from the impact end. Detected impact loading data were stored in a transient memory, and registered on an $X-Y$ pen recorder. An example of an impact-loading curve is illustrated in Fig. 4. The loading frequency is about $67 / \mathrm{min}$.

The characteristics of plastic flow due to cyclic impact blows were evaluated by the decrease in the specimen height, $\Delta H=H_{0}-H_{N}$, and the increase in the impact surface diameter, $\Delta D=D_{N}-D_{0}$, where $H_{N}$ and $D_{N}$ are specimen height and impact surface diameter after the $N$-th blow, respectively, and $H_{0}$ and $D_{0}$ are their initial values. Furthermore, the change in the hardness distribution on the impact surface with the number of loading cycles was measured. The impact surface and subsurface flow structure were observed using SEM and TEM.

\section{3 Low cyclic fatigue test}

The cyclic push-pull loading test was carried out un-

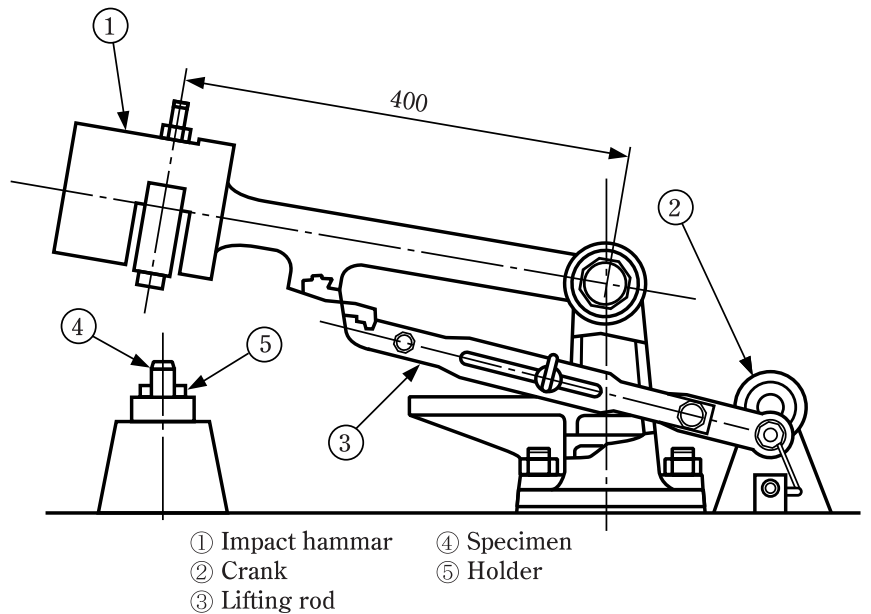

Fig. 3 Impact blow test machine

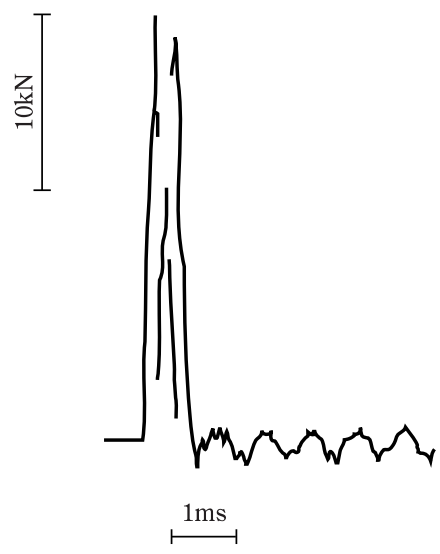

Fig. 4 Impact loading curve

der the following condition; total strain amplitude was constant and the strain rate was $7 \times 10^{-4} / \mathrm{s}$ using an Instron low cycle fatigue test machine. Strains were detected by a clip-on-gauge placed on the section of the specimen with parallel sides.

\section{Experimental results}

\section{1 Cyclic impact blow test}

Fig. 5 shows the relation between $\Delta H$ and the number of impact blows $N$ for various hardness values. In the case of pearlite structure (a), the increase rate of $\Delta H$ 

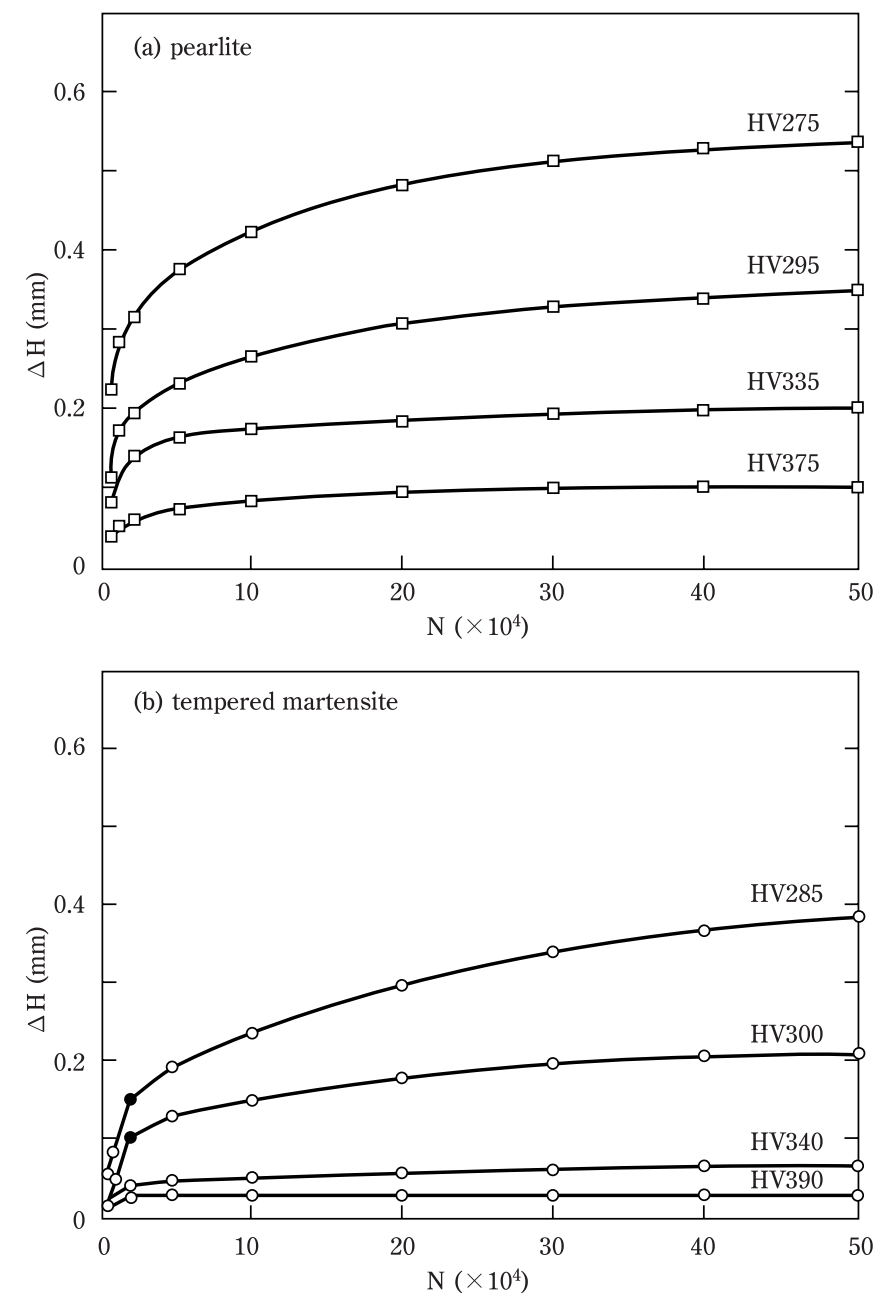

Fig. 5 Relation between $\Delta H$ and the number of impact blows

is large in the early stage, but gradually decreases as $N$ increases and $\Delta H$ finally approaches a constant value. The higher the initial hardness value, the more quickly $\Delta H$ becomes constant. A similar tendency is observed in the case of the tempered martensite (b), but a point of inflection ( in Fig. 5) appears at an early stage. That is, the increase rate of $\Delta H$ increases with $N$ until $N$ reaches nearly 10,000 , but it decreases afterwards with $\Delta H$ approaching a constant value. It seems that the deformation of tempered martensite is considerably smaller than that of pearlite for the same hardness value.

Fig. 6 shows the relation between $\Delta D$ and the number of impact blows $N$. The behavior of $\Delta N$ is similar to that of $\Delta H$, but is more conspicuous. If the hardness exceeds HV300, the increase rate of deformation decreases rapidly and $\Delta D$ soon levels out. In such a case, the final value of $\Delta D$ in pearlite is almost the same as that in tempered martensite. Fig. 7 shows the change in diametrical hardness distribution on the impact surface with $N$. In the case of the pearlite structure (a), hardness increases monotonically as $N$ increases. The increase rate is large in the early stages and decreases gradually. In contrast, in the case of the tempered martensite (b), hardness decreases with $N$ in the early stage, but when $N$ exceeds nearly 10,000 , it increases conversely. The value of $N$ at which this change occurs almost coincides with $N$ corre-
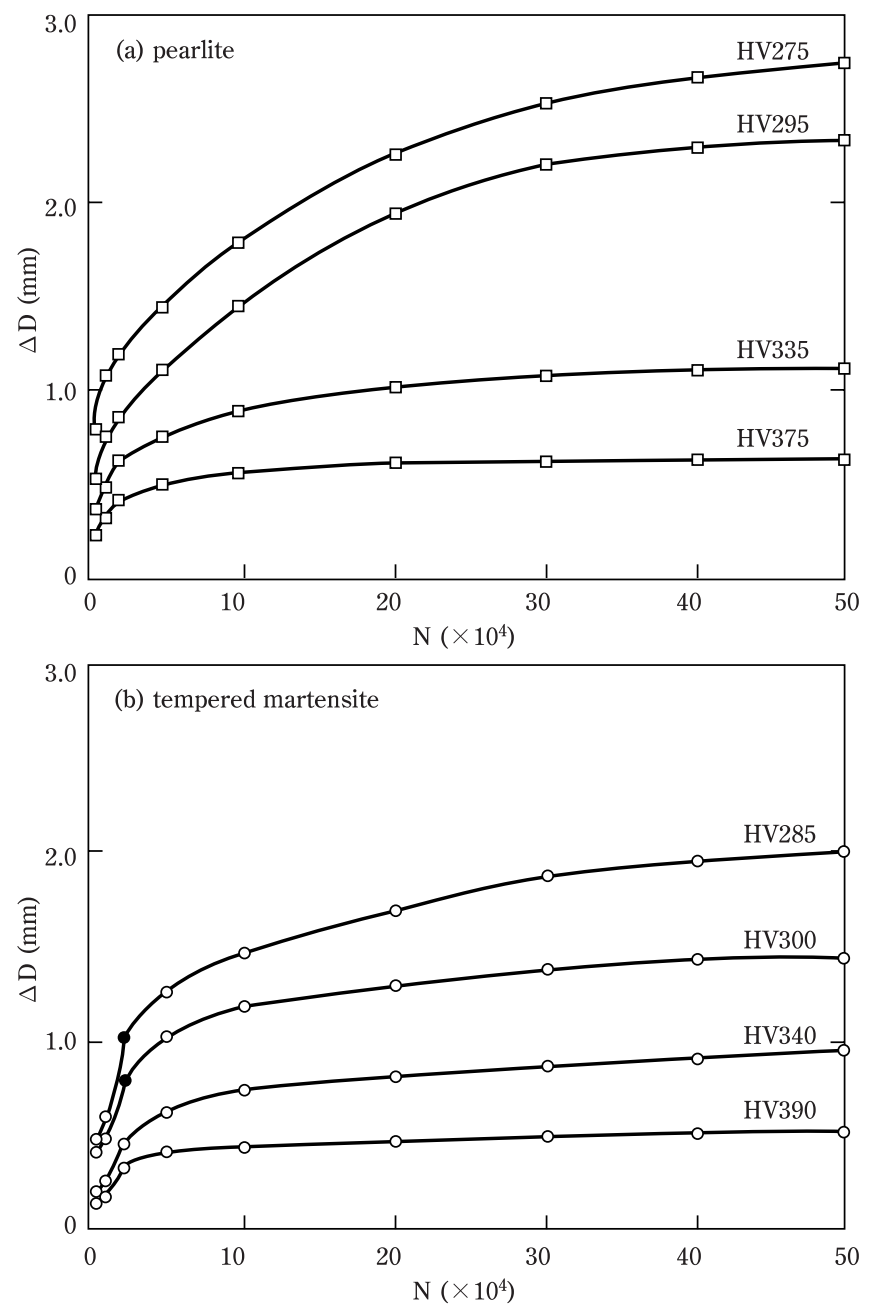

Fig. 6 Relation between $\Delta D$ and the number of impact blows
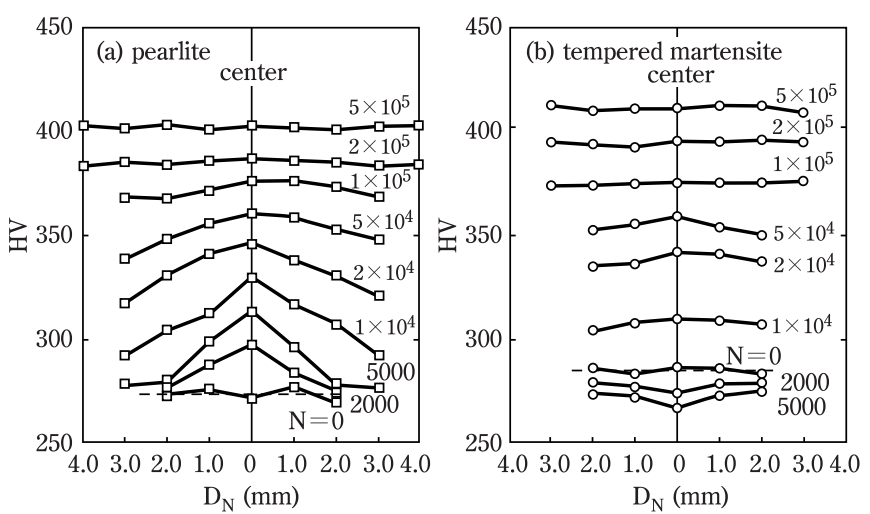

Fig. 7 Changes in hardness on the impact surface with the number of impact blows

sponding to the point of inflection in Figs.5 (b) and 6(b). The final hardness depends only on the initial hardness value regardless of the structure. In the early stages, a sharp peak of hardening or softening appears at the center of the specimen. But it tends to gradually become constant all over the surface with the increase in $N$.

\section{2 Low cycle fatigue test}

Determining the cyclic stress-strain curve from the 
medium pearlite

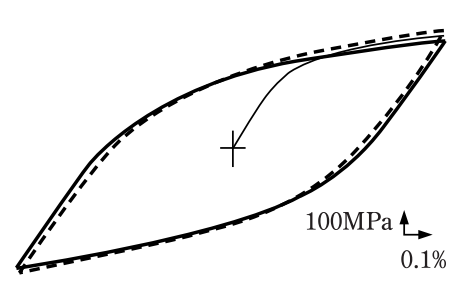

1st cycle tempered martensite

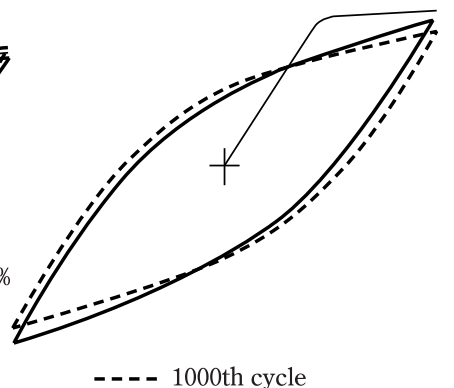

Fig. 8 Stress-strain hysteresis loops
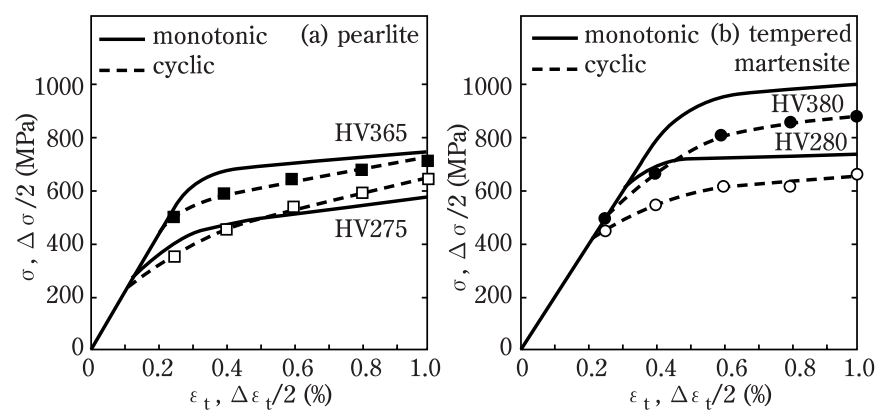

Fig. 9 Monotonic and cyclic stress-strain curves

multiple step tests consists of cycling a single specimen at several levels of strain amplitude. The number of cycles taken at each level should be large enough for the hysteresis loop to become stable but small enough to avoid any serious fatigue damage. With the stable hysteresis loops superimposed, the cyclic stress-strain curve is obtained by drawing a smooth curve connecting the tips of the loops ${ }^{1}$.

Fig. 8 shows the first and the 1,000 th stress-strain hysteresis loops for both medium pearlite and tempered martensite. In medium pearlite, the flow stress increases as the number of cycles increases, but decreases remarkably in tempered martensite. Fig. 9 shows cyclic stressstrain curves obtained from hysteresis loops and monotonic stress-strain curves. In fine pearlite (HV365), only cyclic softening occurs in this strain range. Maximum softening occurs at a $0.4 \%$ strain amplitude, and softening decreases as the strain amplitude further increases. However, in medium pearlite (HV275), cyclic softening occurs at strain amplitudes less than $0.5 \%$, while cyclic hardening occurs at those above $0.5 \%$. Even in fine pearlite $^{2)}$, if the strain amplitude increases further, a cyclic hardening will occur. On the other hand, in tempered martensite (b), only cyclic softening occurs at every hardness level. Softening increases with higher initial hardness values.

When Figs. 9 (a) and 9(b) are compared, it can be seen that softening in tempered martensite is more conspicuous than that in pearlite. Monotonic stress-strain curves are expressed by the equation (1).

$$
\begin{aligned}
& \sigma=K \varepsilon_{p}^{n} \quad \cdots \cdots \cdots \cdots \cdots \cdots \cdots \cdots \cdots \cdots \cdots \cdots \cdots \cdots(1) \\
& \text { where, } \sigma \text { : stress } \\
& \varepsilon_{p}: \text { plastic strain }
\end{aligned}
$$

\begin{tabular}{|c|c|c|c|c|c|c|}
\hline & $\begin{array}{c}\sigma_{y} \\
(\mathrm{MPa})\end{array}$ & $\begin{array}{c}\sigma_{y}^{\prime} \\
(\mathrm{MPa})\end{array}$ & $n$ & $\begin{array}{c}K \\
(\mathrm{MPa})\end{array}$ & $n^{\prime}$ & $\begin{array}{c}K^{\prime} \\
(\mathrm{MPa})\end{array}$ \\
\hline $\begin{array}{l}\text { Pearlite } \\
\text { (HV365) }\end{array}$ & 690 & 600 & 0.12 & 1390 & 0.25 & 2670 \\
\hline $\begin{array}{l}\text { Pearlite } \\
\text { (HV275) }\end{array}$ & 475 & 445 & 0.15 & 1225 & 0.28 & 2515 \\
\hline $\begin{array}{c}\text { Tempered } \\
\text { martensite } \\
(\mathrm{HV} 380)\end{array}$ & 970 & 740 & 0.07 & 1485 & 0.20 & 1824 \\
\hline $\begin{array}{c}\text { Tempered } \\
\text { martensite } \\
(\text { HV290) }\end{array}$ & 720 & 550 & 0.11 & 1288 & 0.17 & 1760 \\
\hline $\begin{aligned} \sigma_{y} & : \text { Monotor } \\
\sigma_{y}^{\prime} & : \text { Cyclic y } \\
n & : \text { Monotor } \\
n^{\prime} & : \text { Cyclic s } \\
K & : \text { Monotor } \\
K^{\prime} & : \text { Cyclic s }\end{aligned}$ & $\begin{array}{l}\text { c yield s } \\
\text { ld stress } \\
\text { c strain } \\
\text { ain hard } \\
\text { c streng } \\
\text { ength co }\end{array}$ & $\begin{array}{l}\text { ress at } 0 \\
\text { at } 0.2 \% \\
\text { ardenin } \\
\text { ning ex } \\
\text { h coeffic } \\
\text { fficient }\end{array}$ & $\begin{array}{l}\% \text { off } \\
\text { ffset } \\
\text { expon } \\
\text { nent } \\
\text { nt }\end{array}$ & & & \\
\hline
\end{tabular}

Table 3 Monotonic and cyclic mechanical properties
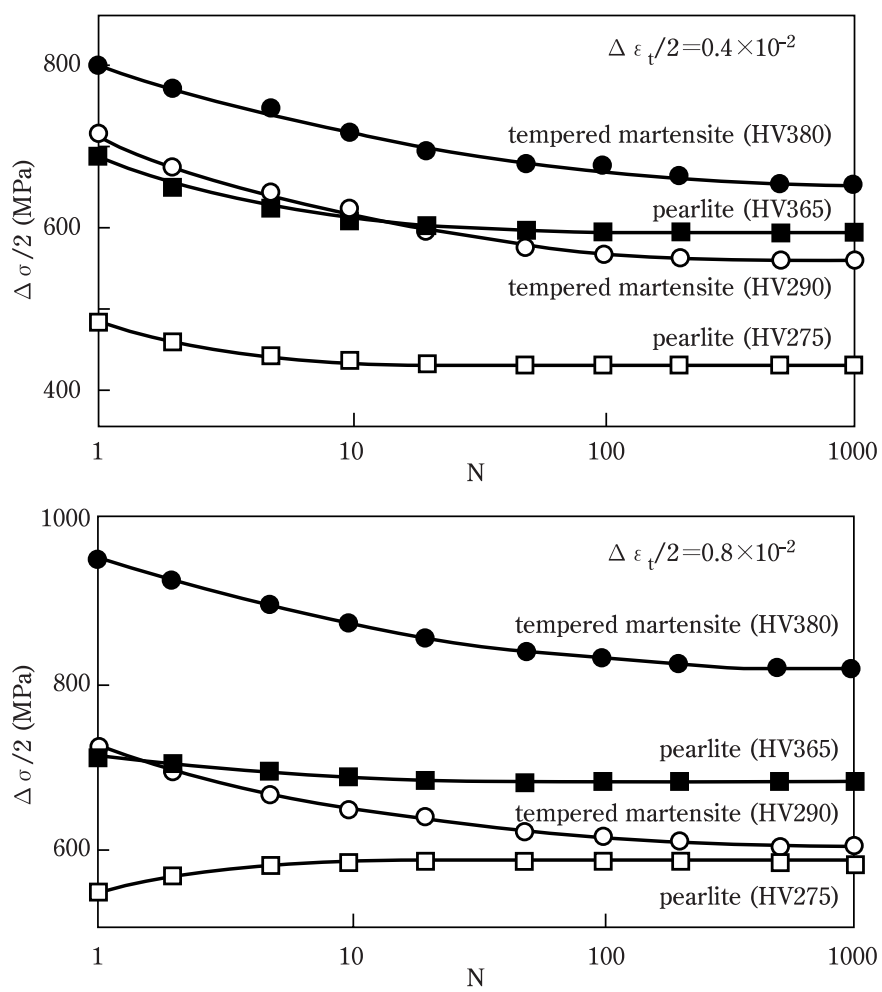

Fig. 10 Relation between stress amplitude $\Delta \sigma / 2$ and number of cycles $N$

$K$ : monotonic strength coefficient

$n$ : monotonic strain hardening exponent.

And cyclic stress-strain curves by

$$
\Delta \sigma / 2=K^{\prime}\left(\Delta \varepsilon_{p} / 2\right)^{n^{\prime}}
$$

where, $\Delta \sigma / 2$ : stress amplitude

$\Delta \varepsilon_{p} / 2 \quad$ : plastic strain amplitude

$K^{, p} \quad$ : cyclic strength coefficient

$n^{\prime} \quad$ : cyclic strain hardening exponent.

Applying these equations to the results obtained gives the parameters shown in Table 3. For the same hardness value, the $0.2 \%$ offset yield stress in tempered martensite is larger than that in pearlite. The cyclic strain hardening exponent $n^{\prime}$ is larger than the monotonic strain 
hardening exponent $n$ in every specimen. Fig. 10 shows representative relations between the stress amplitude $\Delta \sigma / 2$ and the number of cycles $N$. In the case of a $0.4 \%$ strain amplitude, only a cyclic softening occurs in each structure and hardness. But in the case of a $0.8 \%$ strain amplitude, a cyclic hardening occurs only in medium pearlite (HV275). The stress amplitude in pearlite becomes stable more quickly than that in tempered martensite.

\section{Discussion}

\subsection{Deformation behavior under cyclic loading}

Fig. 11 shows the relation between $\Delta D$ and the hardness at the center of the impact surface, which can be roughly classified into three stages; the initial transition stage( I in Fig. 11), rapid hardening stage( II ) and gradual hardening stage( III ). In the case of pearlite, hardening occurs in stage I . While for tempered martensite, softening occurs in this stage. Stage III appears clearly in pearlite, and especially in medium pearlite (HV275). In stage III , many cracks like those shown in Fig. 12 (a) are observed on the impact surface of a pearlite specimen. On the other hand, they are rarely observed in tempered martensite. It seems that the stage III appearing in pearlite is caused by cracking and crack opening.

Fig. 13 shows changes in the deformation increment $\Delta d,(=\mathrm{d}(\Delta D) / \mathrm{d} N)$, obtained from the data shown in Fig. 6. Klesnil et al. ${ }^{3)}$ have obtained similar results from low cycle push-pull loading at a constant stress ampli-

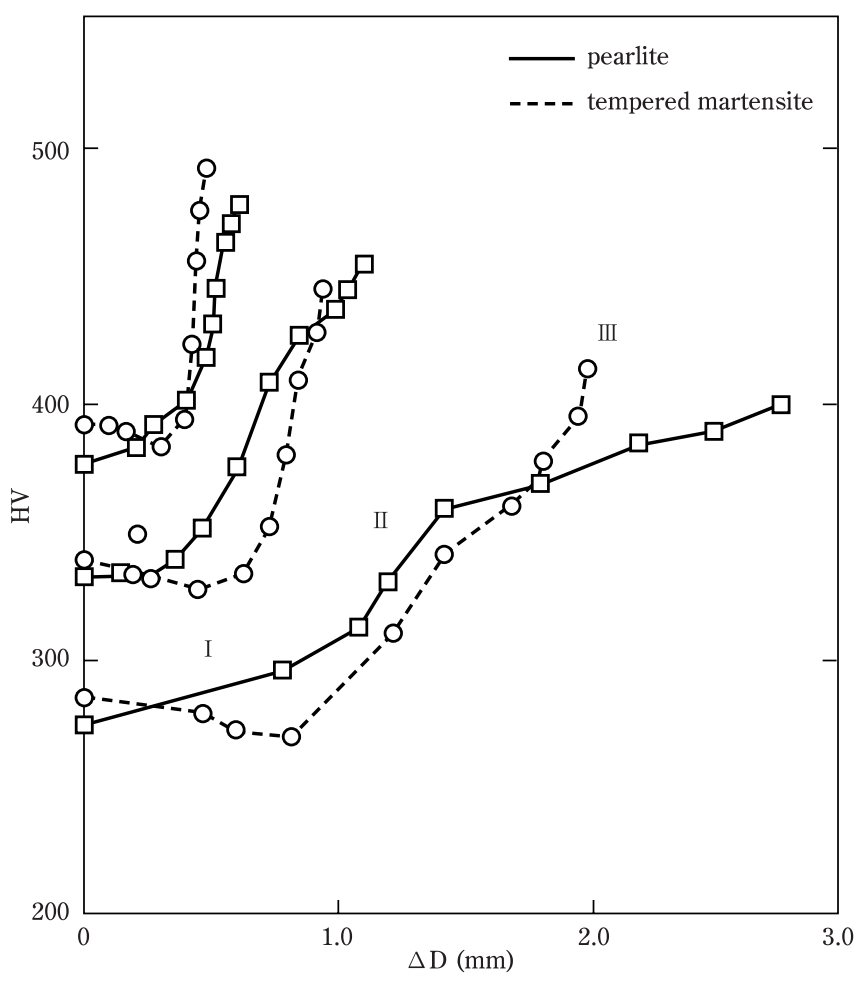

Fig. 11 Relation between $\Delta D$ and the hardness at the center of the impact surface
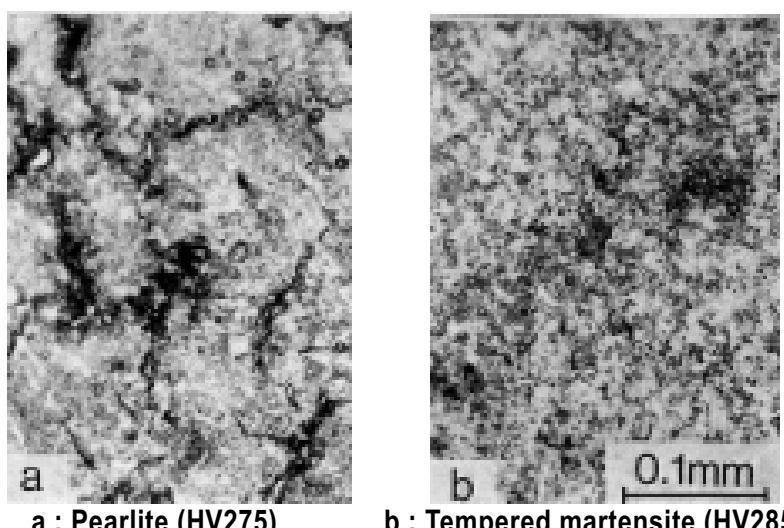

a : Pearlite (HV275)

b : Tempered martensite (HV285)

Fig. 12 Cracks on the impact surface

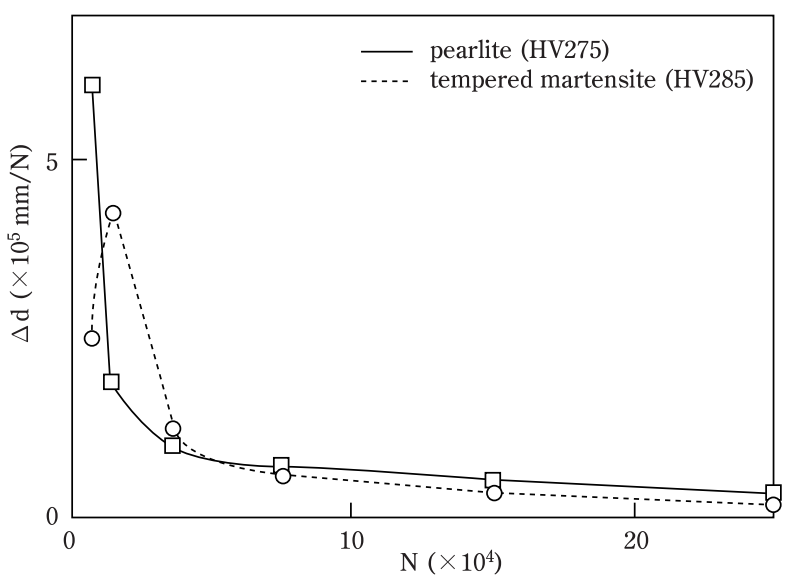

Fig. 13 Relation between $\Delta d$ and $N$

tude. That is, depending on the micro-structure, only cyclic hardening occurs or cyclic softening turns to hardening as $N$ increases. These facts suggest that the deformation behavior in steel under cyclic impact blows and under low cycle fatigue can be thought to be essentially identical. In the impact blow tests, results indicating monotonic hardening in pearlite, and an initial softening stage followed by a subsequent hardening in tempered martensite have been obtained. On the other hand, the low cycle fatigue tests have revealed softening stage at a small strain amplitude followed by subsequent hardening at a large one in pearlite, and monotonic softening in tempered martensite. These contradictory results can be explained as follows: in the case of the impact blow test, the loading is compression only, and this induces localization of softening and hardening at the end surface, as shown in Fig. 7, while in the case of low cycle fatigue, push-pull loading causes uniform softening and hardening over the length of the specimen. Furthermore, the strain amplitude and the number of cycles are quite different in the two tests. Consequently, softening in an early cyclic stage can not be easily observed in the impact blow tests. According to the results of low cycle fatigue tests (Table 3), if the initial hardness value is about the same, both of the monotonic and cyclic yield stress are much larger in tempered martensite than in pearlite. So the final deformation (see Figs.5 and 6) is smaller in tempered martensite than in pearlite even though the former shows noteworthy softening. 


\subsection{Structural change under cyclic loading}

Figs. 14 and 15 show the subsurface microstructures and dislocation structures, respectively. The dislocation
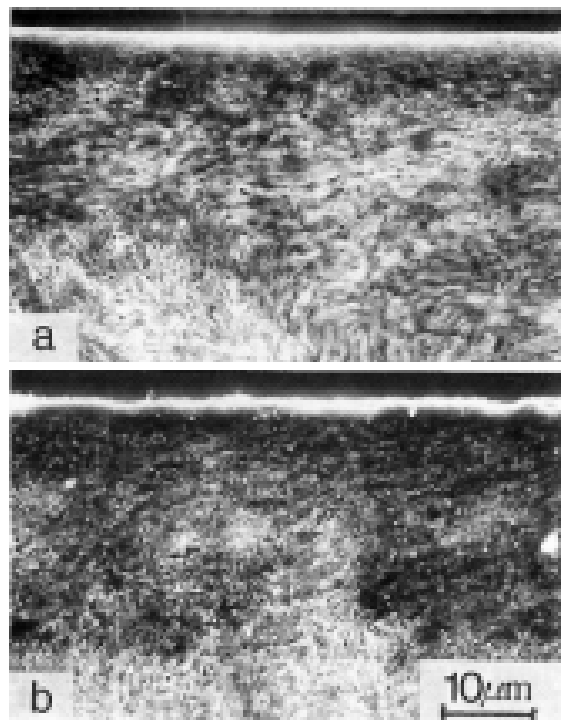

impact

surface

a : Pearlite (HV275)

b : Tempered martensite (HV285)

Fig. 14 Subsurface structure observed by SEM near the impact end of the specimen structure depends on the distance from the impact surface. In the case of pearlite, the microstructure is heavily deformed in a region just under the impact surface (Fig. 15 (a)). In this region, the dislocations were localized in the ferrite causing the cementite to crack $(\Rightarrow)$. However, the cracking did not extend over the whole colony. In the region (Fig. 15 (b)), cell structures developed without any cementite cracking, but it is more difficult to detect them in fine pearlite. In the inner region far from the impact surface (Fig. 15 (c)), a few cell structures are recognized in medium pearlite, but only isolated dislocations can be seen in fine pearlite. In the case of tempered martensite, increases in dislocation density and dislocation tangling take place in the heavily deformed region (Fig. 15 (a)), while a uniform distribution of isolated dislocations in the inner region takes place in martensite laths. Such structural changes can be considered to correspond to deformation stages shown in Fig. 11. Namely, the deformation behaviors in the regions (a), (b) and (c) correspond to the stages III, II and I, respectively. So the deformation process can be explained as follows:

In the pearlite structure, dislocations are rearranged into cell structures in ferrite lamellae at stage I, and hardening occurs rapidly due to dislocation tangling at stage II. As a strain further increases, cementite cracking occurs locally leading afterward to micro-cracking.

On the other hand, in the tempered martensite structure, cyclic softening occurs due to generated mobile dis-
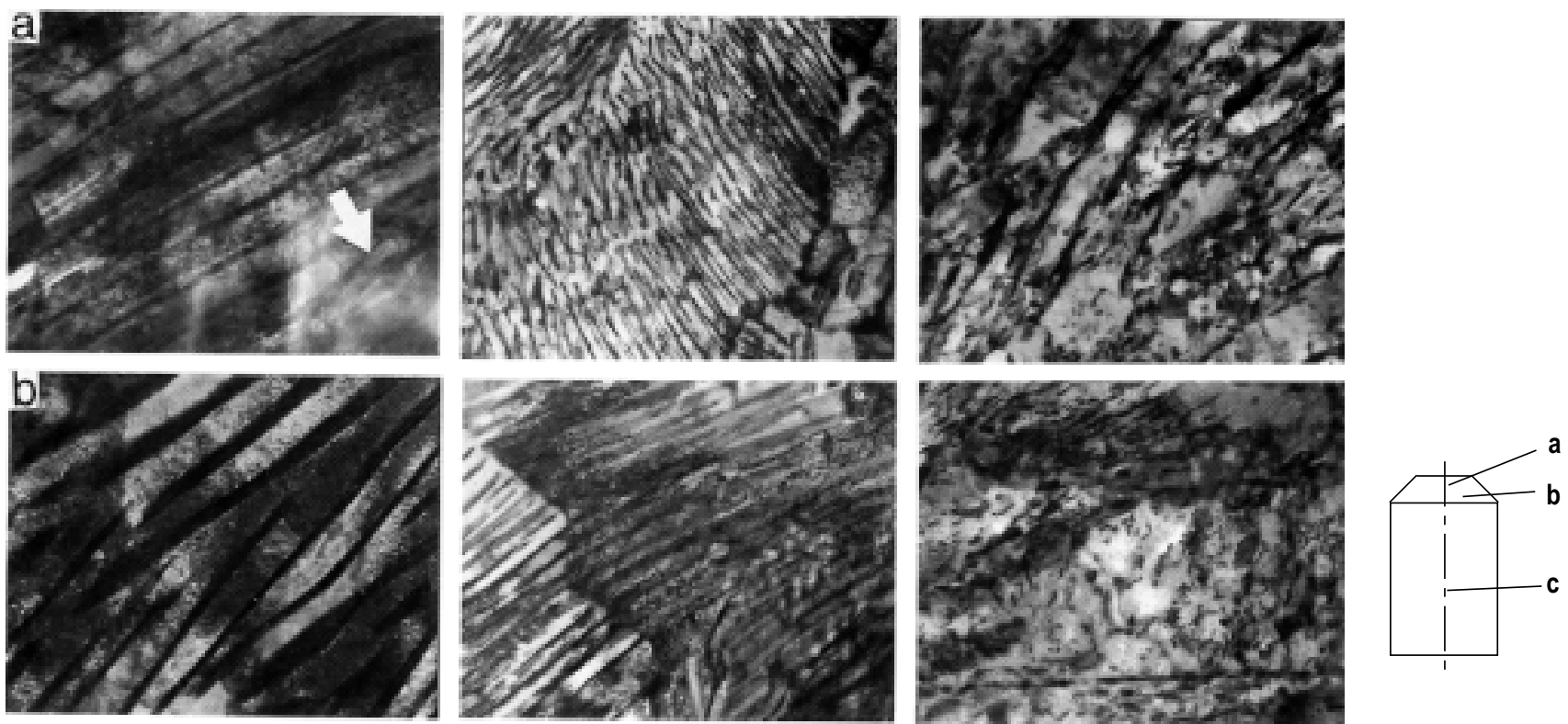

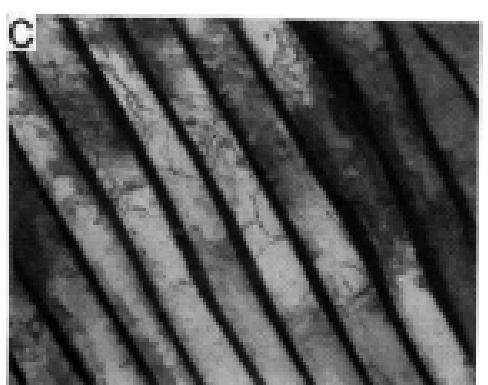

pearlite (HV275)

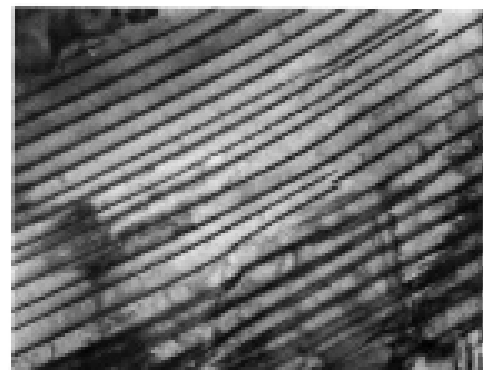

fine pearlite (HV375)

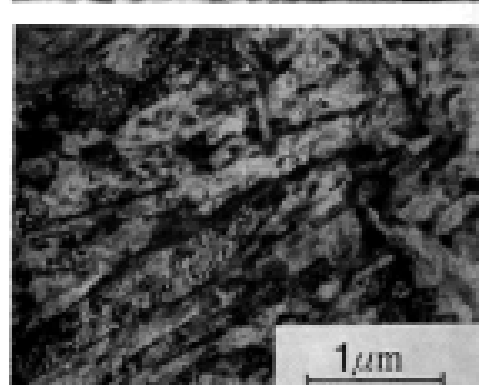

tempered martensite (HV390)

Fig. 15 Dislocation structure in several portions of specimens 
locations and the annihilation of dislocations at stage I, because both dislocation density and hardness are relatively high before impact blows. As strain increases, dislocation blocking due to tangling becomes predominant following the intensive hardening at stage II . However, even more straining can not induce cementite cracking because of the globular structure. So the final deformation is smaller in tempered martensite than in pearlite. From these results, it can be said that tempered martensite structure is superior to pearlite in the effect of preventing plastic deformation of rail by impact blows. However, what causes damage to rails is not only plastic deformation and cracking in the heads but also wear, shelling, etc. Pearlite structure is prominently effective to prevent wear on rail surface ${ }^{4)}$. So the $\mathrm{EH}$ rails should not necessarily be rejected for use, and the optimum structure of rail must be sought from all angles.

\section{Conclusions}

Cyclic impact blow tests and low cycle fatigue tests were performed using $0.75 \% \mathrm{C}$ rail steels with different hardness values and structures. The results are summarized as follows.

(1) The deformation caused by cyclic impact blows is larger in pearlite than that in tempered martensite. In the case of tempered martensite, cyclic softening occurs at an early deformation stage. In contrast to this, only hardening occurs in the case of pearlite. At a stage of heavier deformation, many cracks appear on the impact surface in pearlite, while there is no such cracking in tempered martensite.

(2) In low cycle fatigue tests, cyclic softening occurs at a small strain amplitude in pearlite structure. As the strain amplitude increases, softening turns to hardening. This transition tends to occur at a smaller strain amplitude in medium pearlite than in fine pearlite. On the other hand, in tempered martensite, it seems that only a cyclic softening occurs and more extensively than is observed in pearlite.

(3) The dislocation structures formed under cyclic impact blows depend on the distance from the impact surface and correspond to the structural changes seen under cyclic plastic straining. This fact suggests that the fatigue behavior in cyclic impact blow tests can be roughly estimated by using low cycle fatigue tests.

\section{References}

1) Raske, D. T. and Morrow, J.: "Manual on Low Cycle Fatigue Testing", ASTM Spec. Tech. Publ. p.465, 1969

2) Sunwoo, H., Fine, M.E., Meshii, M. and Stone, D. H.: "Cyclic Deformation of Pearlitic Eutectoid Rail Steel", Metall. Trans. A, 13A, pp.2035-2047, 1982

3) M. Klesnil, M. Holzmann and P. Rys: "Some aspects of the fatigue process in low-carbon steel", J. Iron Steel Inst., 203, pp.47- 53 , 1965

4) M. Akama and S. Matsuyama: "Wear Characteristics of Wheel and Rail Steels", J. JSLE Int. Ed., 8, pp.7580,1987 